\title{
Conference Paper \\ Overview on the Pointwise Constrained Liapunov Vectorial Convexity Theorem
}

\author{
Clara Carlota, Sílvia Chá, and António Ornelas \\ Cima-ue, Rua Romão Ramalho 59, 7000-671 Évora, Portugal \\ Correspondence should be addressed to Clara Carlota; ccarlota@uevora.pt
}

Received 17 May 2013; Accepted 18 June 2013

Academic Editors: B. Datta, G. S. F. Frederico, N. Martins, D. F. M. Torres, and A. J. Zaslavski

This Conference Paper is based on a presentation given by Clara Carlota and Sílvia Chá at “The Cape Verde International Days on Mathematics 2013" held from 22 April 2013 to 25 April 2013 in Praia, Cape Verde.

Copyright (C) 2013 Clara Carlota et al. This is an open access article distributed under the Creative Commons Attribution License, which permits unrestricted use, distribution, and reproduction in any medium, provided the original work is properly cited.

In applications of the Calculus of Variations, Optimal Control and Differential Inclusions, very important real-life problems are nonconvex vectorial and subject to pointwise constraints. The classical Liapunov convexity theorem is a crucial tool allowing researchers to solve nonconvex vectorial problems involving single integrals. However, the possibility of extending such theorem so as to deal with pointwise constraints has remained an open problem for two decades, in the more realistic case using variable vectorial velocities. We have recently solved it, in the sense of proving necessary conditions and sufficient conditions for solvability of such problem. A quick overview of our results is presented here, the main point being that, somehow, convex constrained nonuniqueness a.e. implies nonconvex constrained existence.

\section{Introduction}

Given any $A C$ (absolutely continuous) function $\bar{x}:[a, b] \rightarrow$ $\mathbb{R}^{n}$ whose derivatives may be written as convex combinations of $m$ velocities $v^{j}(\cdot)$, with coefficients $\bar{\lambda}^{j}(\cdot)$,

$$
\bar{x}(t)=\bar{x}(a)+\int_{a}^{t} \sum_{j=1}^{m} \bar{\lambda}^{j}(\tau) v^{j}(\tau) d \tau
$$

by the 1940 classical theorem of Liapunov [1], on convexity of the range of vector measures, one may partition the interval $[a, b]$ into disjoint measurable subsets, having characteristic functions $\chi_{E^{j}}(\cdot)$, such that the new bang-bang function

$$
\widehat{x}(t):=\bar{x}(a)+\int_{a}^{t} \sum_{j=1}^{m} \chi_{E^{j}}(\tau) v^{j}(\tau) d \tau
$$

again satisfies the same boundary conditions:

$$
\widehat{x}(a)=\bar{x}(a), \quad \widehat{x}(b)=\bar{x}(b) .
$$

Here we present a simplified overview of the main results of our recent paper [2], which has improved this Liapunov theorem by again allowing the fulfilment of the boundary conditions (3) - on the global range of this vector measurebut now under an extra pointwise space constraint:

$$
\langle\widehat{x}(t), \omega\rangle \leq\langle\bar{x}(t), \omega\rangle \quad \forall t \in[a, b],
$$

for any $\omega \in \mathbb{R}^{n}$ which one desires to fix once for all, for example $\omega=(1,0, \ldots, 0)$.

Our results have thus generalized (to any spacedimension $n>1$ and any number $m \geq 2$ of velocities $v^{j}(\cdot)$ in (1)) the result of Amar and Cellina [3, Lemma 3.4] (valid for $n=1$ and $m=2$ ). They also generalized, to variable vector velocities, the result of [4] dealing with constant vector velocities.

The many potential applications of our result can be easily guessed by looking at all the applications of the Liapunov convexity theorem to nonconvex differential inclusions, calculus of variations, and optimal control which have been published along the last 3 decades, in particular those due to the last author of this paper and collaborators.

Indeed, our motivation to invest such efforts, in trying to solve this difficult problem, has been precisely the need we felt for disposing of the pointwise constrained Liapunov 
tool in order to unblock our road to solve some important nonconvex vectorial pointwise constrained problems dealing with (single or multiple) integrals of the calculus of variations and optimal control.

\section{Main Function Spaces and Aim}

In order to state the results of the reviewed paper, we start from the space of absolutely continuous vector functions,

$$
\mathscr{X}:=W^{1,1}\left([a, b], \mathbb{R}^{n}\right),
$$

fix $m$ integrable vector velocities

$$
v^{1}(\cdot), \ldots, v^{m}(\cdot) \in L^{1}\left([a, b], \mathbb{R}^{n}\right),
$$

fix any $\bar{x}(\cdot)$ having derivatives in the convex hull "co" of these velocities, that is,

$$
\begin{gathered}
\bar{x}(\cdot) \in \mathscr{X}^{\mathrm{co}}, \\
\mathscr{X}^{\mathrm{co}}:=\left\{x(\cdot) \in \mathscr{X}: x^{\prime}(t) \in \operatorname{co}\left\{v^{1}(t), \ldots, v^{m}(t)\right\} \text { a.e. }\right\},
\end{gathered}
$$

and consider the associated nonconvex differential inclusion

$$
\begin{aligned}
& x^{\prime}(t) \in\left\{v^{1}(t), \ldots, v^{m}(t)\right\} \text { a.e., } \\
& x(a)=\bar{x}(a), \quad x(b)=\bar{x}(b),
\end{aligned}
$$

and its convexified, or relaxed, differential inclusion

$$
\begin{aligned}
& x^{\prime}(t) \in \operatorname{co}\left\{v^{1}(t), \ldots, v^{m}(t)\right\} \text { a.e., } \\
& x(a)=\bar{x}(a), \quad x(b)=\bar{x}(b) ;
\end{aligned}
$$

together with their solution sets

$$
\begin{aligned}
& \bar{X}^{\text {co }}:=\{x(\cdot) \in \mathscr{X}: x(\cdot) \text { solves }(10)\}, \\
& \bar{X}^{\text {true }}:=\{x(\cdot) \in \mathscr{X}: x(\cdot) \text { solves }(9)\} .
\end{aligned}
$$

Fix, moreover, any "direction" $\omega \in \mathbb{R}^{n}$, for example $\omega=(1,0, \ldots, 0)$, and consider the corresponding constrained solution set:

$$
\bar{X}_{-}^{\mathrm{co}}:=\left\{x(\cdot) \in \bar{X}^{\mathrm{co}}:\langle x(t), \omega\rangle \leq\langle\bar{x}(t), \omega\rangle \forall t \in[a, b]\right\}
$$

(constrained relaxed solutions),

where $\langle\cdot, \cdot\rangle$ is the inner product in $\mathbb{R}^{n}$.

Then the aim of this paper is to establish necessary conditions and sufficient conditions for nonemptiness of the class

$$
\bar{X}_{-}^{\text {true }}:=\bar{X}^{\text {true }} \cap \overline{\mathscr{X}}_{-}^{\text {co }}
$$

(constrained true solutions).

\section{The Geometric Control Problem Viewpoint}

The problem we are considering may also be seen as a geometric control problem as follows. Taking, for example, $|\omega|=1$, we are given an $A C$ factor $\bar{h}(\cdot) \in W^{1,1}([a, b])$, a hyperplane $\bar{H}(t)$ orthogonal to $\omega$, namely

$$
\bar{H}(t):=\left\{S \in \mathbb{R}^{n}:\langle S-\omega \bar{h}(t), \omega\rangle=0\right\}, \quad t \in[a, b],
$$

moving along the $\omega$-direction with relaxed velocities

$$
\bar{h}^{\prime}(t) \in \operatorname{co}\left\{\left\langle v^{1}(t), \omega\right\rangle, \ldots,\left\langle v^{m}(t), \omega\right\rangle\right\} \quad \text { a.e. on }[a, b],
$$

and an $A C$ point $\bar{x}(t)$ moving inside $\bar{H}(t)$ with relaxed velocities

$$
\bar{x}^{\prime}(t) \in \operatorname{co}\left\{v^{1}(t), \ldots, v^{m}(t)\right\} \quad \text { a.e. on }[a, b] ;
$$

and we wish to find an $A C$ point $\widehat{x}(t)$ starting from the same initial point, $\widehat{x}(a)=\bar{x}(a) \in \bar{H}(a)$, moving with true velocities

$$
\hat{x}^{\prime}(t) \in\left\{v^{1}(t), \ldots, v^{m}(t)\right\} \quad \text { a.e. on }[a, b],
$$

and reaching exactly the same final endpoint, $\widehat{x}(b)=\bar{x}(b) \epsilon$ $\bar{H}(b)$, under the extra constraint of never crossing the moving hyperplane $\bar{H}(t)$.

The first result we have obtained says that the final hyperplane $\bar{H}(b)$ is always easily attained. Namely, setting

$$
\begin{aligned}
& \bar{X}_{\omega}:=\{x(\cdot) \in \mathscr{X}: x(a)=\bar{x}(a),\langle x(b), \omega\rangle=\langle\bar{x}(b), \omega\rangle\}, \\
& \bar{X}_{\omega}^{\mathrm{co}}:=\left\{x(\cdot) \in \bar{X}_{\omega}: x^{\prime}(t) \in \operatorname{co}\left\{v^{1}(t), \ldots, v^{m}(t)\right\} \text { a.e. }\right\}, \\
& \bar{X}_{\omega}^{\text {true }}:=\left\{x(\cdot) \in \bar{X}_{\omega}: x^{\prime}(t) \in\left\{v^{1}(t), \ldots, v^{m}(t)\right\} \text { a.e. }\right\},
\end{aligned}
$$

then our first (trivial) vectorial extension (see [2, Th. 1.1]) of the scalar pointwise-constrained Liapunov result of [3] consists in the following.

Theorem 1 (existence of minimal, and maximal, constrained true weak solutions).

$$
\begin{aligned}
& \forall \bar{x}(\cdot) \in \mathscr{X}^{c o} \quad \forall \omega \in \mathbb{R}^{n} \quad \exists x_{\omega}^{\min }(\cdot) \in \bar{X}_{\omega}^{\text {true }} \\
& \exists x_{\omega}^{\max }(\cdot) \in \bar{X}_{\omega}^{\text {true }}: \\
& \quad\left\langle x_{\omega}^{\min }(\cdot), \omega\right\rangle \leq\langle x(\cdot), \omega\rangle \leq\left\langle x_{\omega}^{\max }(\cdot), \omega\right\rangle \quad \forall x(\cdot) \in \overline{\mathscr{X}}_{\omega}^{c o} .
\end{aligned}
$$

However, while the final hyperplane $\bar{H}(b)$ can always be reached, the exact final target $\bar{x}(b)$ inside $\bar{H}(b)$ is not always attainable; so that the real challenge, in this research, has been how to reach, more specifically, $\bar{x}(b)$. 


\section{Our General $m \times n$ Pointwise Constrained Liapunov Convexity Theorem}

In order to state the conditions of solvability for the main problem of reaching the exact aimed final endpoint $\bar{x}(b)$, consider a new subspace of (12) given by

$$
\overline{\mathscr{X}}_{--}^{\mathrm{co}}:=\left\{x(\cdot) \in \overline{\mathscr{X}}_{-}^{\mathrm{co}}:\langle x(t), \omega\rangle\left\langle\langle\bar{x}(t), \omega\rangle \text { a.e. in } \mathcal{O}_{x}\right\},\right.
$$

where $\mathcal{O}_{x}$ is an adequate open set which, to simplify exposition, in this overview will be the simplest possible choice:

$$
\sigma_{x}=(a, b)
$$

(see our paper [2] for more general definitions of this set). Then the main result (see [2, Theorems 1.2 and 1.4]) of our research is as follows.

Theorem 2 (existence of constrained true solutions).

$$
\overline{\mathscr{X}}_{--}^{c o} \neq \emptyset \Longrightarrow \bar{X}_{-}^{\text {true }} \neq \emptyset, \quad \forall m \geq 2 \quad \forall n \geq 1 \text { in (6); }
$$

or, more precisely, if there exists at least one function in $\bar{X}_{-}^{c o}$ then $\bar{x}(\cdot)$ may be uniformly approximated by a $\omega$-increasing sequence of true solutions:

$$
\begin{aligned}
& \exists\left(\hat{x}^{k}(\cdot)\right) \subset \bar{x}^{\text {true }}:\left(\hat{x}^{k}(\cdot)\right) \longrightarrow \bar{x}(\cdot) \text { and } \\
& \left(\left\langle\hat{x}^{k}(\cdot), \omega\right\rangle\right) \nearrow\langle\bar{x}(\cdot), \omega\rangle \text {, uniformly. }
\end{aligned}
$$

Thus, somehow, convex constrained nonuniqueness a.e. implies nonconvex constrained existence. Moreover, we have proved the following characterization (see [2, proof of Theorem 1.2]):

Theorem 3 (necessary and sufficient condition for existence of constrained true solutions). One has

$$
\begin{aligned}
& \bar{X}_{-}^{\text {true }} \neq \emptyset \text { if and only if } \exists \tilde{x}(\cdot) \in \bar{X}_{-}^{c o} \text { such that } \\
& \tilde{x}^{\prime}(t) \in\left\{v^{1}(t), \ldots, v^{m}(t)\right\} \text { for a.e. } t \in[a, b] \backslash \mathcal{O}, \\
& \text { where } \mathcal{O}:=\{t \in(a, b):\langle\tilde{x}(t), \omega\rangle<\langle\bar{x}(t), \omega\rangle\} .
\end{aligned}
$$

\section{Nonemptiness of $\bar{X}_{--}^{\mathrm{co}}$ in the Special $2 \times 2$ Case}

Although (22) provides a nice and simple theoretical sufficient condition for existence of constrained true solutions, in practical applications it is not at all clear, a priori, whether the above class $\bar{X}_{--}^{\text {co }}$, in (22) and (20) is, or is not, empty.

In this section, we present our first contribution towards clarifying such issue, namely through necessary conditions, and sufficient conditions, to have $\bar{X}_{--}^{\text {co }}$ nonempty and to have $\bar{X}_{--}^{\text {co }}$ empty. However, for simplicity, we will restrict ourselves to the most basic nontrivial framework: just $m=2$ velocities to be given in (6), in space-dimension $n=2$; and with difference between these two velocities having second coordinate writable as a factor $f(t)$ times the first one. Thus we assume given

$$
\begin{gathered}
v^{1}(\cdot), v^{2}(\cdot) \in L^{1}\left([a, b], \mathbb{R}^{2}\right), \\
\left(v^{2}-v^{1}\right)(t)=v(t)(1, f(t)) \text { a.e. },
\end{gathered}
$$

for some $v(\cdot) \geq 0$ a.e. in $L^{1}(a, b)$ and some $f(\cdot)$ in

$$
\mathscr{F}:=\left\{f(\cdot) \in L^{1}(a, b):(f \cdot v)(\cdot) \in L^{1}(a, b)\right\} .
$$

While this may sound, at first sight, like a very very special situation, in reality such very basic framework is precisely what we need for our applications, of the constrained Liapunov convexity Theorem 2, to the Calculus of Variations involving vector-valued functions in competition towards minimization of nonconvex single or multiple integrals, under state and gradient pointwise constrained Lagrangians.

Again for simplicity, let us assume

$$
\omega=(1,0),
$$

and associate to each $f(\cdot) \in \mathscr{F}$ the new class of functions

$$
\begin{gathered}
\mathscr{G}_{f}:=\left\{g(\cdot) \in W_{0}^{1,1}([a, b]): g(\cdot) \neq \equiv 0,\right. \\
\left.\left(f \cdot g^{\prime}\right)(\cdot) \in L^{1}(a, b)\right\} .
\end{gathered}
$$

We begin by presenting a necessary condition (see [2, Theorem 2.1]).

Theorem 4 (necessary condition in $2 \times 2$ case). For each given $f(\cdot)$ and $\bar{x}(\cdot)$, as in (25), (26), and (7), so that

$$
\begin{aligned}
& \exists \bar{\lambda}(\cdot) \in L^{\infty}(a, b): \bar{\lambda}(t) \in[0,1] \text { a.e., } \\
& \bar{x}^{\prime}(t)=v^{1}(t)+\bar{\lambda}(t) v(t) \cdot(1, f(t)) \text { a.e., }
\end{aligned}
$$

it turns out that

$$
\begin{gathered}
\text { if } \overline{\mathscr{X}}_{-}^{c o} \backslash\{\bar{x}(\cdot)\}\left(\text { resp. } \bar{X}_{--}^{c o}\right) \text { is nonempty then } \\
\exists \bar{g}_{f}(\cdot) \in \mathscr{G}_{f}: \bar{g}_{f}(\cdot) \geq 0\left(\text { resp. } \bar{g}_{f}(\cdot)>0 \text { a.e. }\right) \\
\quad \int_{a}^{b} f(t) \bar{g}_{f}^{\prime}(t) d t=0 \\
-[v \cdot(1-\bar{\lambda})](t) \leq \bar{g}_{f}^{\prime}(t) \leq[v \cdot \bar{\lambda}](t) \text { a.e. }
\end{gathered}
$$

In particular, such necessary condition yields simple counterexamples (independent of $\bar{x}(\cdot)$ ) illustrating the necessity of including some extra hypothesis, for example nonemptiness-as in (22) - of the set $\bar{X}_{--}^{\text {co }}$, in order to guarantee solvability of our pointwise constrained Liapunov problem. Namely,

$$
\begin{aligned}
& f(\cdot) \text { a.e. strictly monotone } \\
& \left.\quad \Longrightarrow \overline{\mathscr{X}}_{-}^{\mathrm{co}} \backslash\{\bar{x}(\cdot)\}=\emptyset \quad \text { (see }(12)\right) ;
\end{aligned}
$$


or, as an alternative,

$$
\begin{gathered}
f(\cdot) \text { a.e. monotone and } \int_{a}^{b}\left|f^{\prime}(t)\right| d t>0 \\
\Longrightarrow \bar{X}_{--}^{\text {co }}=\emptyset \quad(\text { see }(20))
\end{gathered}
$$

(or, more precisely, $\exists$ monotone $\check{f}(\cdot)=f(\cdot)$ a.e. and $\left.\int_{a}^{b}\left|\check{f}^{\prime}(t)\right| d t>0\right)$.

On the other hand, in order to reach a sufficient condition guaranteeing $\overline{\mathscr{X}}_{--}^{\text {co }} \neq \emptyset$, let us associate to each $f(\cdot)$ as in (25) and (26) and to each $d \in\{1,2, \ldots\}$ a

$$
d \times d \text {-symmetric matrix } M_{f}^{d}
$$

whose entries

$$
\begin{aligned}
M_{f}^{i j}:= & (-1)^{k} \frac{(k+1) !}{i ! j !} \\
& \times\left[(k+2) f^{(-k-2)}(b)-(b-a) f^{(-k-1)}(b)\right],
\end{aligned}
$$

for $k:=i+j$ and $i, j \in\{0,1, \ldots, d-1\}$, are linear combinations of pairs of iterated indefinite integrals of $f(\cdot)$ :

$$
\begin{gathered}
f^{(0)}(t):=f(t) \\
f^{(-k-1)}(t):=\int_{a}^{t} f^{(-k)}(\tau) d \tau \quad \text { for } k=0,1,2, \ldots
\end{gathered}
$$

Then, given any reference relaxed solution $\bar{x}(\cdot)$ hence a corresponding $\bar{\lambda}(\cdot)$, as in (7) and (29), we finally present our main result on the special $2 \times 2$ case (see [2, Theorem 2.5]).

Theorem 5 (Sufficient Condition in $2 \times 2$ case). Under (25), (26), (27), and (29), assume the matrix $M_{f}^{d}$ has,

$$
\begin{aligned}
& \text { for some } i \in\{1,2, \ldots\}, \quad M_{f}^{i i} \cdot M_{f}^{00} \leq 0 \\
& \left(\text { or, more generally, } M_{f}^{i i} \cdot M_{f}^{00} \leq\left(M_{f}^{0 i}\right)^{2}\right)
\end{aligned}
$$

or, still more generally, $M_{f}^{d}$ has

$$
\text { eigenvalues } \beta_{-}, \beta_{+} \text {with } \beta_{-} \cdot \beta_{+} \leq 0 \text {, }
$$

and the following inequalities hold true: (see (26) and (29))

$$
-\operatorname{ess} \inf [v \cdot(1-\bar{\lambda})]([\mathrm{a}, \mathrm{b}])<0<\operatorname{ess} \inf [v \cdot \bar{\lambda}]([\mathrm{a}, \mathrm{b}]) \text {. }
$$

Then

$$
\exists \widetilde{x}(\cdot) \in \bar{x}_{--}^{\text {co }} \text { having } \tilde{x}^{\prime}(\cdot):=\bar{x}^{\prime}(\cdot)-g^{\prime}(\cdot) \cdot(1, f(\cdot)) \text {, }
$$

with a $2 d$-degree polynomial $g(\cdot)>0$ a.e. having $g(a)=0=$ $g(b)$ and satisfying (30).

\section{Open Problems and Challenges}

While we have thus obtained nice and useful research results, the truth is that each conquest always raises new and interesting questions whose answer is unknown, hence opening new challenges for future researches.

In particular, we are already working on extending the results here presented, namely to optimal control problems steered by linear differential inclusions of first order, which poses some nice challenges, and of higher order, posing still harder challenges. Another related question is the application of these results to optimal control problems involving minimization of pointwise-constrained multiple integrals, which was in reality our original motivation.

We would welcome brave Masters or Ph.D. students interested in following and contributing to our researches in this field.

\section{Acknowledgments}

The research leading to this paper was performed at: Cimaue (Math Research Center of Universidade de Évora, Portugal) with financial support from the research project PEstOE/MAT/UI0117/2011, FCT (Fundação para a Ciência e a Tecnologia, Portugal).

\section{References}

[1] A. A. Liapounoff, "Sur les fonctions-vecteurs complètement additives," Izvestiya Akademii Nauk SSSR, vol. 4, pp. 465-478, 1940.

[2] C. Carlota, S. Chá, and A. Ornelas, "A pointwise constrained version of the Liapunov convexity theorem for single integrals," Nonlinear Differential Equations and Applications, vol. 20, no. 2, pp. 273-293, 2013, Erratum in Nonlinear Differential Equations and Applications, vol. 20, no. 3, pp. 1409, 2013.

[3] M. Amar and A. Cellina, "On passing to the limit for nonconvex variational problems," Asymptotic Analysis, vol. 9, no. 2, pp. 135-148, 1994.

[4] M. Amar and C. Mariconda, "A nonconvex variational problem with constraints," SIAM Journal on Control and Optimization, vol. 33, pp. 299-307, 1995. 


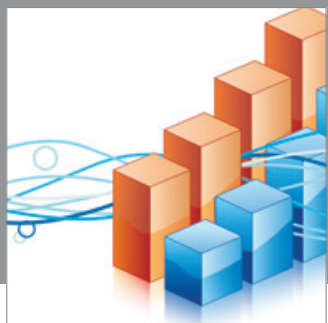

Advances in

Operations Research

mansans

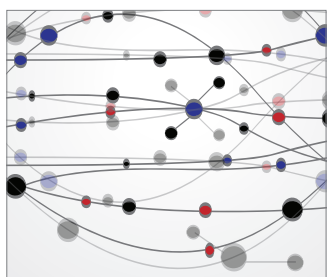

The Scientific World Journal
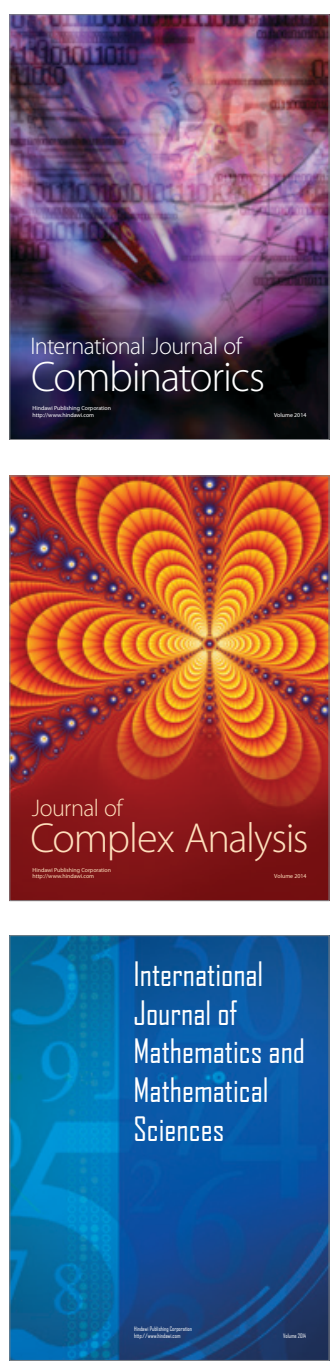
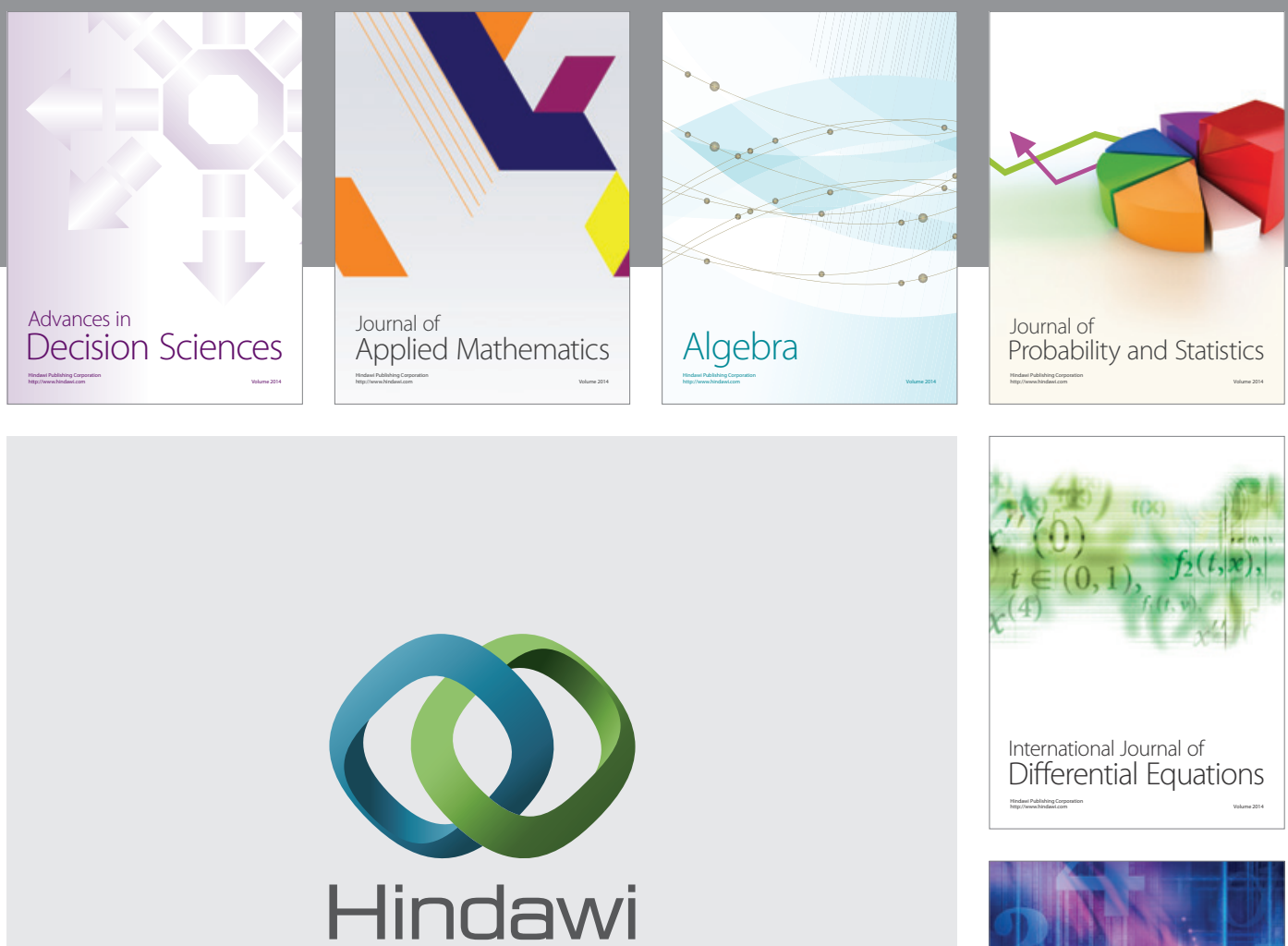

Submit your manuscripts at http://www.hindawi.com
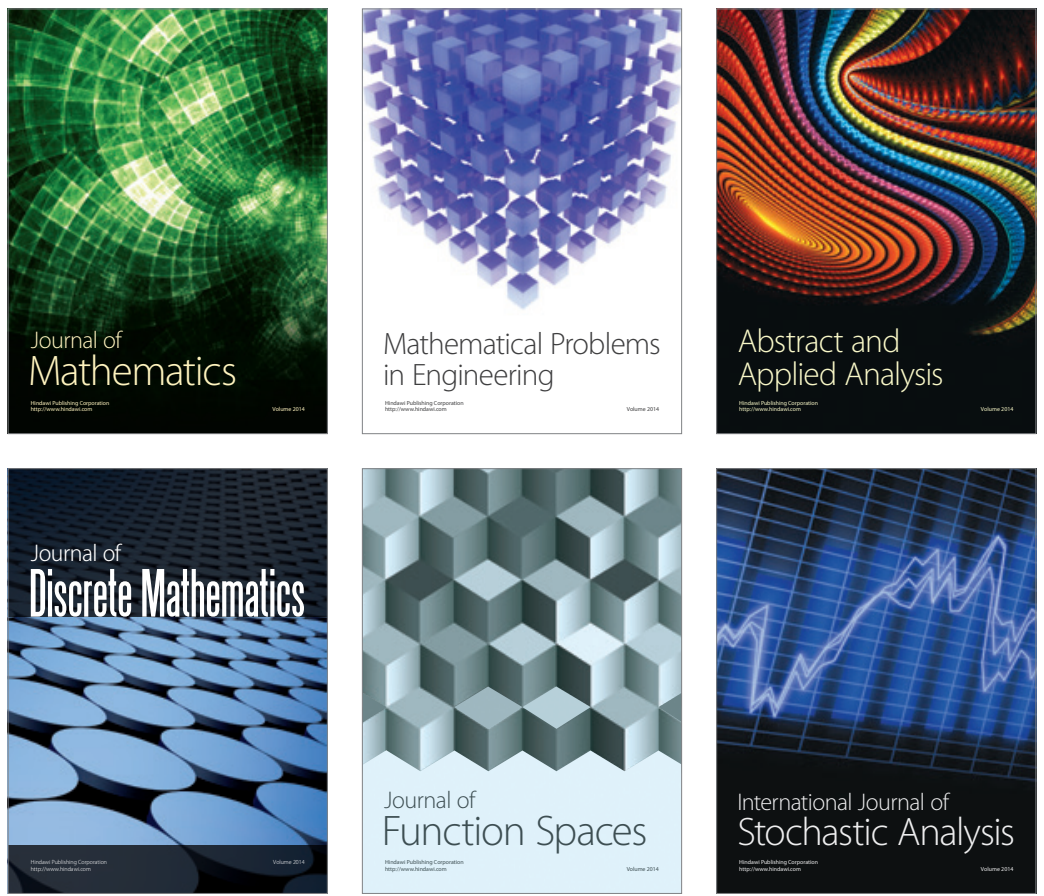

Journal of

Function Spaces

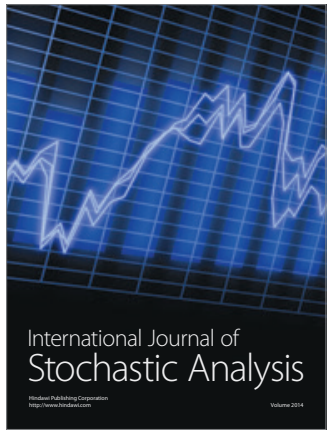

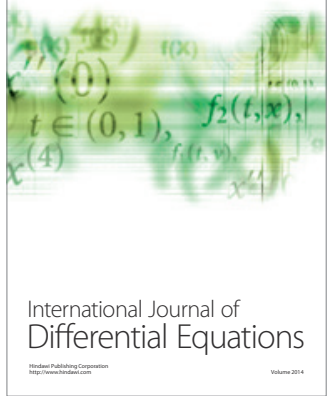
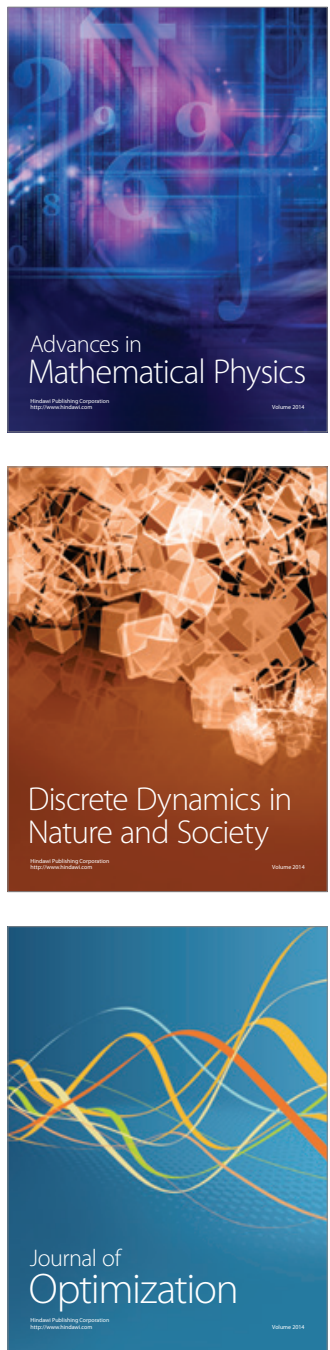